\title{
Low Dose X-Ray Sources and High Quantum Efficiency Sensors: The Next Challenge in Dental Digital Imaging?
}

\author{
Arnav R. Mistry, ${ }^{1}$ Daniel Uzbelger Feldman, ${ }^{1}$ Jie Yang, ${ }^{2}$ and Eric Ryterski ${ }^{3}$ \\ ${ }^{1}$ Department of Endodontology, Temple University Kornberg School of Dentistry, 3223 North Broad Street, \\ Philadelphia, PA 19140, USA \\ ${ }^{2}$ Oral and Maxillofacial Radiology Department, Temple University Kornberg School of Dentistry, 3223 North Broad Street, \\ Philadelphia, PA 19140, USA \\ ${ }^{3}$ E3 Medical, Inc., 941 Garfield Avenue, Louisville, CO 80027, USA \\ Correspondence should be addressed to Daniel Uzbelger Feldman; duzbelger@gmail.com
}

Received 21 August 2014; Revised 10 November 2014; Accepted 17 November 2014; Published 10 December 2014

Academic Editor: Sotirios Bisdas

Copyright (c) 2014 Arnav R. Mistry et al. This is an open access article distributed under the Creative Commons Attribution License, which permits unrestricted use, distribution, and reproduction in any medium, provided the original work is properly cited.

\begin{abstract}
Objective(s). The major challenge encountered to decrease the milliamperes $(\mathrm{mA})$ level in X-ray imaging systems is the quantum noise phenomena. This investigation evaluated dose exposure and image resolution of a low dose X-ray imaging (LDXI) prototype comprising a low mA X-ray source and a novel microlens-based sensor relative to current imaging technologies. Study Design. A LDXI in static (group 1) and dynamic (group 2) modes was compared to medical fluoroscopy (group 3), digital intraoral radiography (group 4), and CBCT scan (group 5) using a dental phantom. Results. The Mann-Whitney test showed no statistical significance $(\alpha=0.01)$ in dose exposure between groups 1 and 3 and 1 and 4 and timing exposure (seconds) between groups 1 and 5 and 2 and 3. Image resolution test showed group $1>$ group $4>$ group $2>$ group $3>$ group 5 . Conclusions. The LDXI proved the concept for obtaining a high definition image resolution for static and dynamic radiography at lower or similar dose exposure and smaller pixel size, respectively, when compared to current imaging technologies. Lower $\mathrm{mA}$ at the X-ray source and high QE at the detector level principles with microlens could be applied to current imaging technologies to considerably reduce dose exposure without compromising image resolution in the near future.
\end{abstract}

\section{Introduction}

With all other technical factors (e.g., kilovolts, distance, time, etc.) held constant, patient radiation dose is directly proportional to the milliamperes $(\mathrm{mA})$. A $50 \%$ reduction in $\mathrm{mA}$ would result in a decrease in radiation dose by $50 \%$ [1]. Previously, the $\mathrm{mA}$ range has not been taken into consideration in any attempt to reduce radiation dose to which dental patients are being exposed [2-6]. The sensitivity of a digital sensor is measured at a constant wavelength in nanometers $(\mathrm{nm})$ on the basis of the detective quantum efficiency (DQE). This value is used primarily to describe imaging detectors in optical imaging and medical radiography [7]. The quantum efficiency $(\mathrm{QE})$ is the ratio of impinging photons on a pixel to the number of collected electrons. The QE of the pixel is equal to the $\mathrm{QE}$ of the complementary metal oxide semiconductor (CMOS) photodiode multiplied for the fill factor of the pixel
[8]. Fluoroscopy is a dynamic X-ray or X-ray movie showing images of video frame rates produced by a low $\mathrm{mA} \mathrm{X}$-ray source and image intensification at the detector level [9]. An image intensifier unit is capable of multiplying 1,000 to 20,000 times, electron-by-electron, of the produced image, therefore increasing the system $\mathrm{QE}$ while allowing dose reduction [10, 11]. Unfortunately, image intensifier units and direct radiography large flat panel detectors have heretofore been too bulky to be used inside the mouth as well as being expensive for a dental setting [12-14]. Another disadvantage of intensifiers is image distortion [15]. With regard to visualization of a stent created from 50 microns $(\mu \mathrm{m})$ diameter wires in flat panel Xray fluoroscopy, for the idealized direct detector, the $100 \mu \mathrm{m}$ pixel size resulted in maximum measured contrast sensitivity. For an idealized indirect detector, with a scintillating layer, the maximal measured contrast sensitivity was obtained at $200 \mu \mathrm{m}$ pixel size [16]. 
The major challenge encountered to decrease the $\mathrm{mA}$ level in X-ray imaging systems is the quantum noise phenomena. In electronic imaging systems such as fluoroscopy or intraoral radiography digital sensors, we find three principal sources of noise. The first and most relevant arises from quantum statistics, in which the discrete nature of the radiographic signal (which often is photon-starved) introduces uncertainty into the image. The second is electronic noise which is generated in the detector or detector electronics. The third is quantization error that occurs in digital electronic imaging systems when the signal is digitized. For a quantum statistics noise limited system, if the number of photons used is quadrupled, the noise in the resultant image should be halved [17-20]. Not only X-ray systems but also digital cameras are noise limited and quantum limited. Randomly spaced speckles, called noise, can appear in digital images. Noise is similar to grain that appears in photos taken with traditional cameras using high International Organization of Standardization (ISO) films. Noise increases in photos taken with a digital camera using a high ISO number. The higher ISO number leads to more noise. When noise is present, image detail and clarity are reduced, sometimes significantly. The ISO level indicates the film and digital camera's sensitivity to light. According to the ANSI/ISO classification, a dental film with raw speed of ISO 29-56 would be classified as E speed, while one with speed of ISO 57-112 would be classified as F speed [21]. Photographic film typically has a QE of much less than 10\% [22]. Current digital cameras have improved their ISO settings which can achieve up to ISO 204,800 [23-25]. To improve the sensitivity or QE of front illuminated charged couple device (CCD) and CMOS image sensors without increasing their pixel size, digital cameras manufacturers apply a thin (0.5-1.0 mm thickness) and inexpensive microlens array to the sensors to reach high ISO levels [26]. The microlens principle was invented in the 17 th century where Hooke and Van Leeuwenhoek developed techniques to make small glass lenses for use with their microscopes [27]. The microlens collects and focuses light that would have otherwise fallen onto the nonsensitive areas of the sensor chip, improving the QE significantly (Figure 1) [28].

Attempts for improving digital sensors QE without compromising the system's noise have been made through the introduction of back illuminated and electron multiplied CCD and CMOS image sensors. Their major drawbacks are complicated manufacturing processes and elevated cost [29, 30]. As a result, the most common used image sensor in dentistry is the front illuminated type [31].

Consequently, a lower milliamperes $(\mathrm{mA})$ setting at the $\mathrm{X}$-ray source and the use of front illuminated sensors with microlens or back illuminated sensors at the detector level for an increased QE that reduces the required radiation dose and sensor pixel size without impacting image quality should have a dramatic positive impact on dental radiology and oral diagnosis. The purpose of this investigation was to prove the concept of radiation exposure reduction and dynamic fluoroscopy feasibility in dentistry without compromising image quality by testing a low-dose X-ray imaging (LDXI) prototype comprising a low-dose $\mathrm{X}$-ray source and a high
QE front illuminated sensor with microlens and comparing it to standard of care in terms of dose exposure in milligrays $(\mathrm{mGy})$ and image resolution in lines per millimeters $(\mathrm{lp} / \mathrm{mm})$ [6].

\section{Materials and Methods}

The Temple University Environmental Health and Radiation Safety Institutional Department approved this study. A LDXI prototype (Real Time Imaging Technologies, LLC, Cleveland, $\mathrm{OH})$ was used. The LDXI was comprised of a 35-80 kilovolt peak $(\mathrm{kVp}), 0.1-0.5 \mathrm{~mA}$ X-ray source $\left(9.95^{\prime} \mathrm{L} \times 5.27^{\prime} \mathrm{W} \times\right.$ $5.35^{\prime} \mathrm{H}$ ) (SourceRay, Bohemia, NY), and a $8^{\prime}$ rectangular collimator (Margraf Dental, Jenkintown, PA) and an X-ray detector utilizing a CMOS front illuminated sensor (EOS 5D Mark III, Canon, Japan) having $36 \times 24 \mathrm{~mm}$ effective area, $6.25 \times 6.25$ um pixel size, 22.3 megapixels resolution, and $49 \%$ [32] QE with microlens and capable of performing up to 30 frames per second (fps) for the dynamic video mode. The CMOS sensor modular transfer function (MTF) was $>30 \%$ at the $18 \mathrm{lp} / \mathrm{mm}$ range. Upon low-pass filter and sensor removal, the scintillator/fiber optic plate (AppliedScintech, UK) was coupled at the sensor [33,34] on top of the microlens [35] with optic glue (BEW Engineering, Ketsch, Germany). The scintillator/fiber optic plate measured $20.8 \times 20.8 \mathrm{~mm}$ and was comprised of a 6 um columnar cesium iodide with thallium (CsI : TI) coating with a MTF curve showing and ultimate resolution $>18 \mathrm{lp} / \mathrm{mm}$ at $60 \mathrm{kVp}$ with $98 \%$ attenuation at $70 \mathrm{kVp}$. Camera software (EOS Utility Ink, Japan) and a kid's watch (Disney Store, Orlando, FL) were used in order to establish and calibrate sensor's ISO settings. Images obtained were raw positive still shots and video. Raw images (A) were converted to negative radiographic images (B) through a software and then cropped (Microsoft Digital Suite 2006 Editor, Microsoft, Redmond, WA) (Figure 2).

Ionization chambers Radcal 9010 (Radcal Corp., Monrovia, CA) and RaySafe Xi (RaySafe, Hopkinton, MA) were used to measure the dose exposure in mGy (obtained through rad formula conversion) received by a patient phantom (DXTTR, RINN, Elgin, IL) in all groups and sixteen dosimeters (Landauer, Inc., Glenwood, IL) were utilized for obtaining the dose equivalent in millisievert $(\mathrm{mSv}$ ) (obtained through rem formula conversion) received by the operator simulated distance at $30 \mathrm{~cm}$ for LDXI and fluoroscopy (Figure 3).

ISO settings were established at ISO 5,000 for group 1 and ISO 12,800 for group 2 and image acquisition was made at $1 / 30$ shutter ( 0.03 seconds). Group 1 was exposed to the LDXI prototype (Real Time Imaging Technologies, LLC, Cleveland, $\mathrm{OH})$ at $0.2 \mathrm{~mA}$ and $80 \mathrm{kVp}$ in static mode during 10 intervals from 0 to 27 continuous seconds with a $6.25 \times 6.25$ um pixel size sensor and $49 \%$ QE. Group 2 was exposed to the LDXI prototype at $0.2 \mathrm{~mA}$ and $80 \mathrm{kVp}$ in the dynamic video mode during 31 intervals from 0 to 300 seconds with a $6.25 \times$ 6.25 um pixel size sensor and 49\% QE. Group 3 was exposed to medical fluoroscopy (GE C-arm OEC 9800, Cleveland, $\mathrm{OH})$ at $0.038 \mathrm{~mA}$ and $55 \mathrm{kVp}$ in 31 intervals from 0 to 300 seconds with a $12.8 \times 12.8$ um pixel size sensor and a $9^{\prime}$ image 


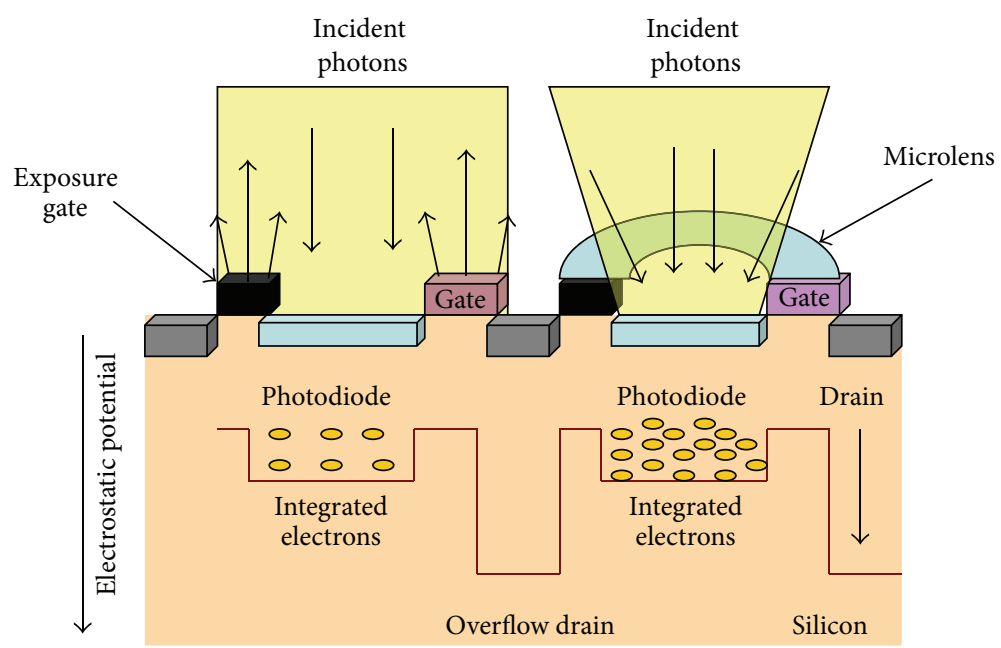

FIGURE 1: CMOS sensor and microlens (right side) architecture scheme.

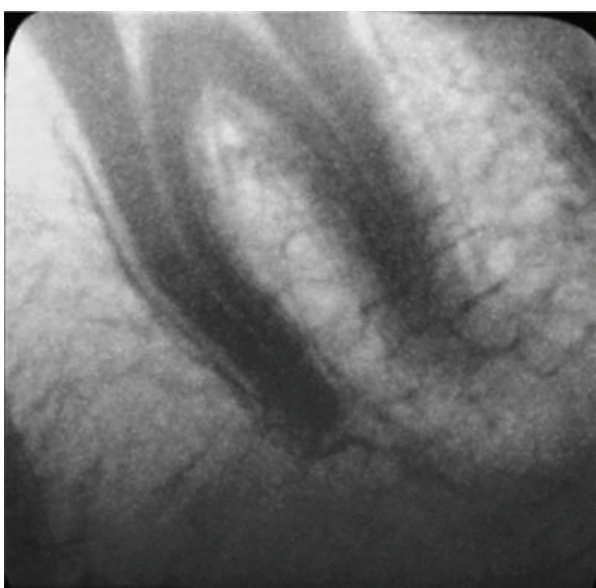

(a)

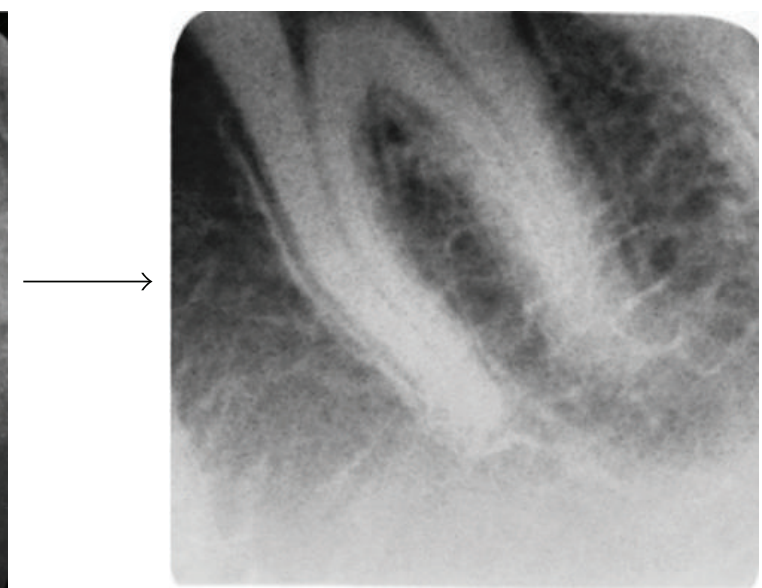

(b)

Figure 2: (a) Molar tooth raw positive image obtained at $0.2 \mathrm{~mA}$ and $1 / 30$ camera shutter (0.03 seconds) with collimation and (b) negative image.

intensifier with 65\% QE at $550 \mathrm{~nm}$ [36]. Group 4 was exposed to digital intraoral radiography (Gendex GX-770, Hatfield, PA/Planmeca Dixi 2 v3, Roselle, IL) at $7 \mathrm{~mA}$ and $70 \mathrm{kVp}$ in 29 intervals from 0 to 1.65 seconds with a $19 \times 19$ um pixel size sensor. Group 5 was exposed to CBCT Scan (iCat Imaging Sciences International Inc., Hatfield, PA) at $5 \mathrm{~mA}$ and $120 \mathrm{kVp}$ from 0 to 26.9 seconds in 28 intervals and several modalities with a $125 \mathrm{um}$ voxel size sensor. Six thermoluminescent Luxel and ten optical stimulated luminescence technology Nanodots dosimeters (Landauer, Inc., Glenwood, IL) were used at the patient phantom, area monitor, and positive and negative controls for the LDXI. After research completion, all the dosimeters were mailed back to Landauer for analysis purposes. Image resolution for the LDXI was measured with a resolution test pattern (Fluke Corp., Cleveland, $\mathrm{OH}$ ) in $\mathrm{lp} / \mathrm{mm}$. An endodontic file size 10 (Dentsply, Maillefer, York, PA) was placed within phantom's tooth number 27 at the radiographic apex for intraoral imaging subjective resolution assessment purposes in which two endodontists and one oral and maxillofacial radiologist from the institution were asked to confirm tooth's working length [6]. Dose exposure measurements and image resolution were calculated for all groups and dosimeters were analyzed.

\section{Results}

The Mann-Whitney test showed no statistical significance ( $\alpha=0.01)$ in dose exposure (mGy) between groups 1 and 3 and 1 and 4 and timing exposure (seconds) between groups 1 and 5 and 2 and 3 (Figure 4).

Dosimeters for the LDXI operator simulated distance and controls did not register significant dose equivalent $(\mathrm{mSv})$.

Image resolution test showed LDXI $1>$ digital intraoral radiography $>$ LDXI $2>$ medical fluoroscopy $>$ CBCT scan (Table 1). 


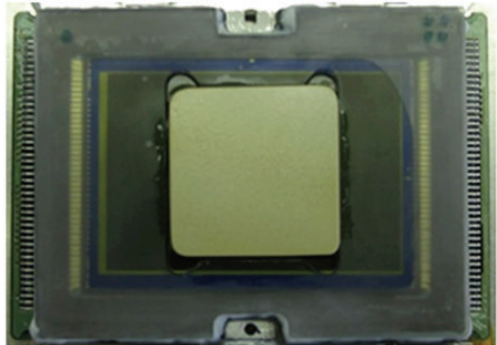

(a)

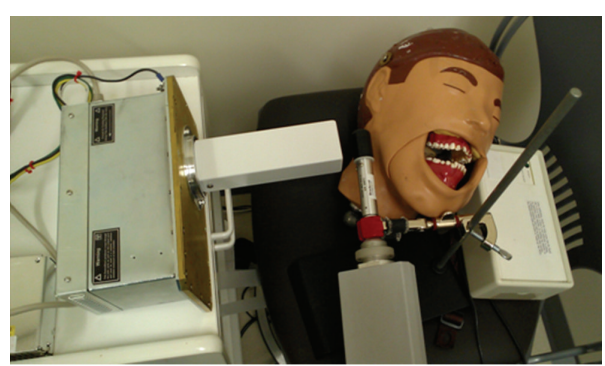

(b)

FIGURE 3: (a) Dental size sensor with microlens coupled with scintillator/FOP (front view) and (b) LDXI prototype testing on the DXTTR phantom at $0.2 \mathrm{~mA}$ and $80 \mathrm{kVp}$.

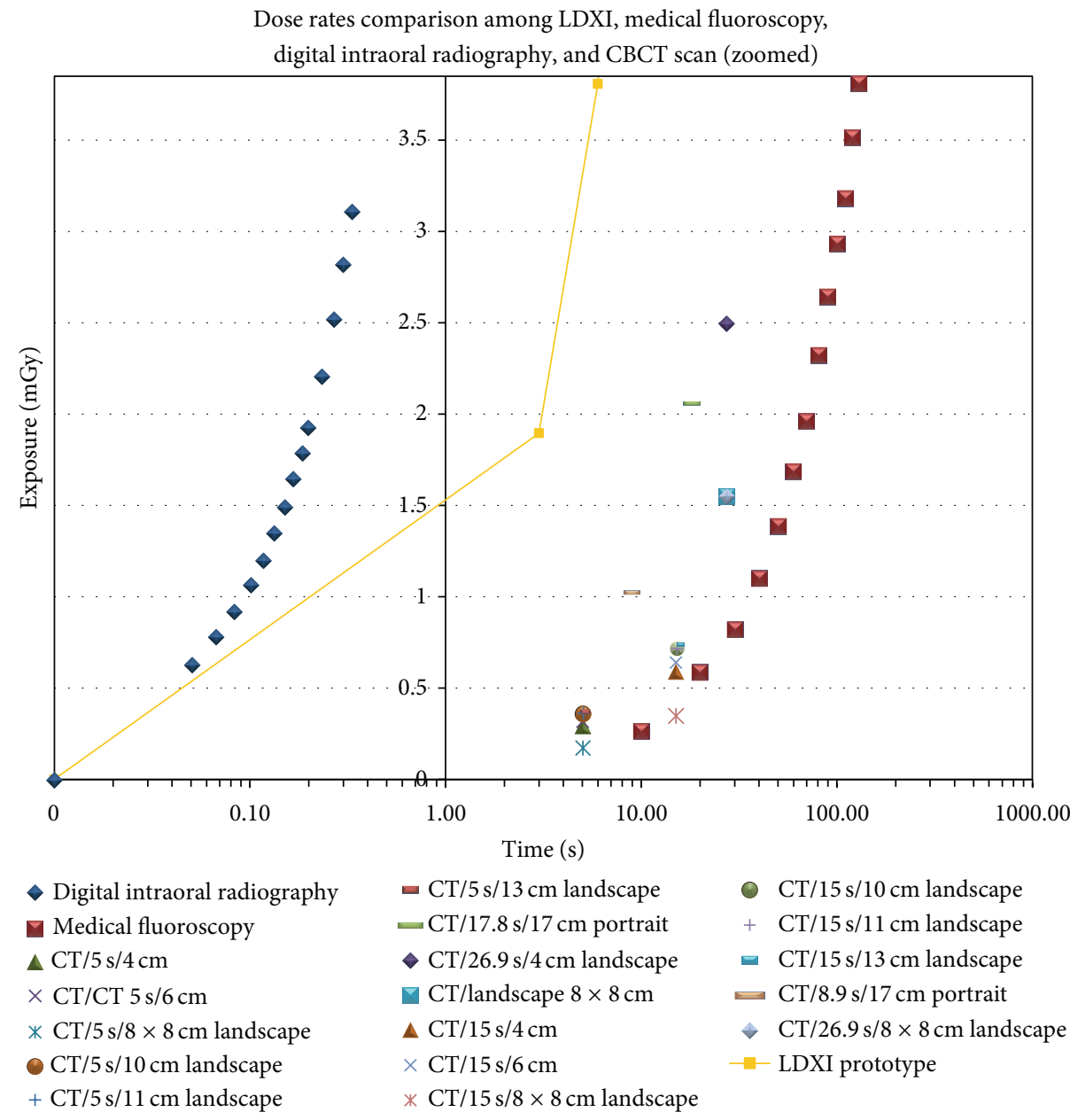

FIGURE 4: Radiation and timing exposures comparison amongst all groups.

File size 10 was observed at the working length as confirmed by two endodontists and one oral and maxillofacial radiologist (Figure 5).

\section{Discussion}

Technical advances enable cameras to better capture the drama of low-light photography/video. As a rule, bigger is indeed better when it comes to the overall dimensions of a sensor because a bigger sensor provides not only more pixels, but also bigger pixels, which more efficiently gather light. However, of all the improvements in the imaging field, perhaps the most notable are not the increases in sensor size but the innovations in increasing a sensor's ability to gather light in low-light situations and to record a wider range of light. Much improved sensors with microlens now allow one to 


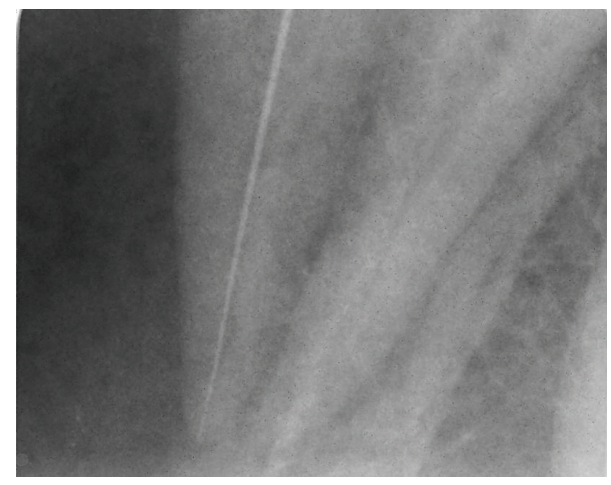

(a)

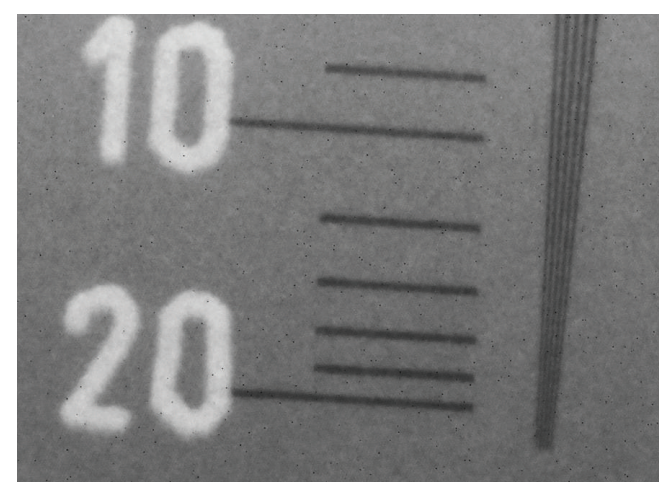

(b)

FIGURE 5: Group 1 LDXI static: (a) endodontic file number 10 at working length and (b) $18 \mathrm{lp} / \mathrm{mm}$.

TABLE 1: $\mathrm{mA}, \mathrm{kVp}$, pixel size settings, and resolution outcome comparison of different devices.

\begin{tabular}{lcccc}
\hline Device type & $\mathrm{mA}$ & $\mathrm{kVp}$ & $\begin{array}{c}\text { Pixel/voxel } \\
\text { size } \\
\text { (um) }\end{array}$ & $\begin{array}{c}\text { Resolution } \\
(\mathrm{lp} / \mathrm{mm})\end{array}$ \\
\hline LDXI 1 (static) & 0.2 & 80 & $6.25 \times 6.25$ & 18 \\
\hline LDXI 2 (dynamic) & 0.2 & 80 & $6.25 \times 6.25$ & 10 \\
\hline $\begin{array}{l}\text { Medical } \\
\text { fluoroscopy (image } \\
\text { intensified) }\end{array}$ & 0.038 & 55 & $12.8 \times 12.8$ & 1.75 \\
\hline $\begin{array}{l}\text { Digital intraoral } \\
\text { radiography }\end{array}$ & 7 & 70 & $19 \times 19$ & 16 \\
\hline CBCT scan & 5 & 120 & 125 & 1.6 \\
\hline
\end{tabular}

obtain detailed low-light images by allowing shots at high ISO levels that previously generated unflattering digital "noise" or graininess in an image at smaller pixel sizes. From an image resolution dental perspective, studies have demonstrated that spatial resolution affects bone loss and caries diagnosis in dentistry. A research undertaken to determine the effect of Xray beam alignment and spatial resolution on quantification of alveolar bone using radiometric techniques concluded that $50 \mathrm{um}$ pixel spatial resolution is apparently superior to 200 um pixel images if radiometric data is to be evaluated [37]. Another study determined that spatial resolution affected radiometric analyses aimed at detecting progressive enamel loss. Cumulative percent histograms shifts associated with the smaller 59 um pixels accounted for $68 \%$ of the variation in weights caused by enamel reduction, whereas the shifts associated with the larger 200 um pixels accounted for $50 \%$. The results indicated that pixel size does affect radiometric determinations of enamel reduction [38, 39]. In summary, the smaller the pixel size and the more pixels are arranged in a sensor, the better the quality of the image that is captured. In this study, microlens also allows decreasing the sensor's pixel size $(6.25 \times 6.25 \mathrm{um})$, therefore increasing image spatial resolution as compared to the larger pixel size of current sensors used in digital intraoral radiography $(19 \times$ $19 \mathrm{um}$ ), CBCT scans (125 um voxel), and medical fluoroscopy
$(12.5 \times 12.5 \mathrm{um}$ for image intensifiers and $100 \times 100 \mathrm{um}$ for flat panel detectors). As compared to similar front illuminated intraoral sensors used in dentistry (DQE 3\% CCD and DQE 18\% CMOS) $[40,41]$ the LDXI prototype has used a large size $(35 \mathrm{~mm})$, full frame, and front illuminated CMOS sensor with a thin microlens array for improved DQE (49\%) directly coupled to the scintillator/fiber optic plate, capable of acquiring low $\mathrm{mA}$ images at ISO 5,000 and ISO 12,800 as well as in dynamic video frame rates. From a radiation exposure perspective, we found that 3.05 and 3.95 seconds of LDXI are comparable to one single shot of 0.2 seconds of digital intraoral radiography and 26.9 seconds $4 \mathrm{~cm}$ landscape single area CBCT scan, respectively. As a result, the LDXI used for 0.2 seconds would reduce $93.4 \%$ dose exposure as compared to digital intraoral radiography used for 0.2 seconds. For a conventional root canal requiring a pre-op, working length, master cone, partial condensation, and final X-ray, the LDXI prototype could be used during 15 seconds without producing more dose exposure due to the addition of microlens for increased QE as compared to digital intraoral radiography. As a result, lower $\mathrm{mA}$ settings (up to $0.2 \mathrm{~mA}$ ) as compared to digital intraoral radiography $(7 \mathrm{~mA})$ could be captured at $18 \mathrm{lp} / \mathrm{mm}$ and $10 \mathrm{lp} / \mathrm{mm}$ image resolution for the static and dynamic modalities, respectively [6]. In addition, an endodontic file size 10 was observed at the working length at the phantom as confirmed by two endodontists and one oral and maxillofacial radiologist from the institution (Figure 5). In addition to root canals, other clinical applications for low dose X-ray sources and high QE image sensors in the static mode would be full mouth X-rays, pre- and postradiographs, bitewings, and panoramic, cephalometric, and CBCT scans for oral diagnosis while the dynamic mode would allow the introduction of fluoroscopy in dentistry for dental implant placement, temporomandibular joint analysis, maxillofacial surgeries, and postplacement. Since the scintillator/fiber optic plate was coupled in the central area of the sensor, the effect of X-rays on the surrounding, residual, and uncovered area caused significant noise causing black spots at the image. In addition, despite the fact that sensor's housing was internally masked against light, light pollution was observed on one corner (Figure 6). 


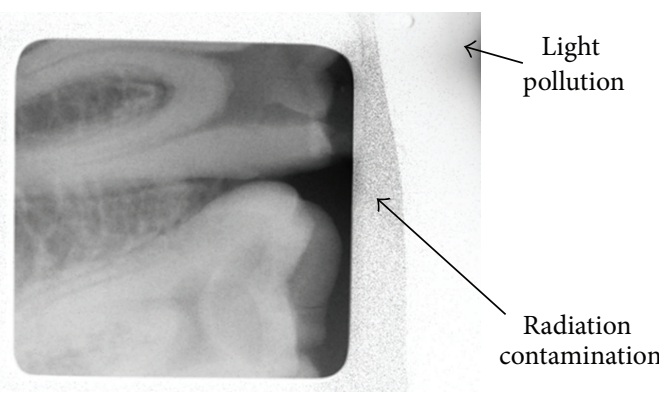

Figure 6: Preventable artifacts obtained in images.

These artifacts should be avoided in future studies by providing complete sensor shielding and a 100\% light proof housing. In addition to further evaluations with higher QE $(>65 \%$ at $550 \mathrm{~nm})$ new generation front illuminated sensors with microlens, the authors recommend testing a back illuminated sensor due to its increased QE as compared to the front illuminated sensor used in this study [14, 35]. Finally, we propose testing the next generation LDXI using head and neck anthropomorphic phantoms following Sections C and D of the FDA guidance for Solid State X-Ray Imaging Devices (MTF, detective quantum efficiency, and signal-tonoise ratio) and compare it to digital intraoral radiography, medical fluoroscopy, and CBCT scan imaging devices.

\section{Conclusions}

The LDXI proved the concept for obtaining a high definition image resolution for static and dynamic radiography at lower or similar dose exposure and smaller pixel size, respectively, when compared to traditional imaging devices. Lower $\mathrm{mA}$ at the X-ray source and high $\mathrm{QE}$ at the detector level principles with microlens could be applied not only to digital intraoral radiography and dental fluoroscopy but also to panoramic, cephalometric, and $\mathrm{CBCT}$ scans devices to considerably reduce X-ray source dose exposure as well as sensor pixel size and more research is recommended to demonstrate this further.

\section{Diclosure}

The paper, or any part of it, has not been submitted or published and will not be submitted elsewhere for publication while being considered by the journal. The research has been shown as a poster presentation at Temple University Kornberg School of Dentistry Research Day and has been presented at the 64th AAOMR Annual Session as an abstract and as part of the 2013 William H. Rollins Award Lecture.

\section{Conflict of Interests}

Jie Yang is a consultant to and Daniel Uzbelger Feldman has equity interest in Real Time Imaging Technologies, LLC. Arnav R. Mistry and Eric Ryterski had no conflict of interests. At the time this study was conducted, Arnav R. Mistry was an endodontology postgraduate student at Temple University Kornberg School of Dentistry.

\section{Acknowledgments}

This study has been possible thanks to the funds received by Real Time Imaging Technologies, LLC (Cleveland, OH), from the Great Lakes Innovation \& Development Enterprise Grant $(\$ 25,000)$ and the Indus Entrepreneurs (TiE) Ohio Business Plan Competition Award $(\$ 2,500)$ as well as by the research team from Temple University Kornberg School of Dentistry Student Research Grant $(\$ 2,000)$.

\section{References}

[1] Q. B. Carroll, Fuchs's Radiographic Exposure and Quality Control, Charles C Thomas Publisher, 7th edition, 2003.

[2] D. Uzbelger, "Comparison between medical fluoroscopy, digital dental imaging and intraoral radiography," Journal of Dental Research, vol. 97, p. 701, 2005.

[3] D. Uzbelger-Feldman, C. Susin, and J. Yang, "The use of fluoroscopy in dentistry: a systematic review," Oral Surgery, Oral Medicine, Oral Pathology, Oral Radiology, and Endodontology, vol. 105, article e61, 2008.

[4] D. Uzbelger Feldman, J. Yang, and C. Susin, "A systematic review of the uses of fluoroscopy in dentistry," The Chinese Journal of Dental Research, vol. 13, no. 1, pp. 23-29, 2010.

[5] D. Uzbelger Feldman, P. M. Panchal, and J. Yang, "Resolution and dose rates comparison among fluoroscopy, digital/filmbased intraoral radiography and CBCT scan," in Proceedings of the 61th Annual Meeting of the American Academy of Oral \& Maxillofacial Radiology, San Diego, Calif, USA, 2010.

[6] D. Uzbelger Feldman, A. Mistry, and J. Yang, "Radiation exposure and image resolution testing of a low-dose X-ray imaging," in Proceedings of the 64th Annual Meeting of American Academy of Oral \& Maxillofacial Radiology, Beverly Hills, Calif, USA, October 2013.

[7] A. R. Cowen, A. G. Davies, and M. U. Sivananthan, "The design and imaging characteristics of dynamic, solid-state, flat-panel $\mathrm{x}$-ray image detectors for digital fluoroscopy and fluorography," Clinical Radiology, vol. 63, no. 10, pp. 1073-1085, 2008.

[8] G. C. Holst and T. S. Lomheim, CMOS/CCD Sensors and Camera Systems, SPIE Press Book, Bellingham, Wash, USA, 2nd edition, 2011.

[9] B. D. Giordano, S. Ryder, J. F. Baumhauer, and B. F. DiGiovanni, "Exposure to direct and scatter radiation with use of mini-carm fluoroscopy," Journal of Bone and Joint Surgery-Series A, vol. 89, no. 5, pp. 948-952, 2007.

[10] J. T. Thirlwall, "The detective quantum efficiency of medical xray image intensifiers," Review of Scientific Instruments, vol. 69, no. 11, pp. 3953-3957, 1998.

[11] S. Hasham, F. D. Burke, S. J. Evans, M. K. Arundell, and D. N. Quinton, "An audit of the safe use of the mini c-arm image intensifier in the out-patient setting," Journal of Hand Surgery, vol. 32, no. 5, pp. 563-568, 2007.

[12] J. A. Seibert, "Flat-panel detectors: how much better are they?" Pediatric Radiology, vol. 36, no. 14, pp. 173-181, 2006.

[13] D. Uzbelger Feldman, "Real Time Imaging Technologies, LLC, Assignee. Apparatus for Diagnosis and/or Treatment in the Field of Dentistry using Fluoroscopic and Conventional Radiography," US Patent 6543936, 2003. 
[14] D. Uzbelger Feldman, "Real Time Imaging Technologies, LLC, assignee. Dental Fluoroscopic Imaging System," US patent 8,430,563, April 2013.

[15] D. P. Chakraborty, "Image intensifier distortion correction," Medical Physics, vol. 14, no. 2, pp. 249-252, 1987.

[16] Y. Jiang and D. L. Wilson, "Optimization of detector pixel size for stent visualization in x-ray fluoroscopy," in Medical Imaging 2004: Image Perception, Observer Performance, and Technology Assessment, vol. 5372 of Proceedings of SPIE, p. 311, May 2004.

[17] B. A. Arnold and P. O. Scheibe, "Noise analysis of a digital radiography system," American Journal of Roentgenology, vol. 142, no. 3, pp. 609-613, 1984.

[18] D. W. Mah, J. A. Rowlands, and J. A. Rawlinson, "Measurement of quantum noise in fluoroscopic systems for portal imaging," Medical Physics, vol. 23, no. 2, pp. 231-238, 1996.

[19] M. Hensel, T. Pralow, and R. R. Grigat, "Modeling and realtime estimation of signal-dependent noise in quantum-limited imaging," in Proceedings of the 6th WSEAS International Conference on Signal Processing, Robotics and Automation (ISPRA '07), World Scientific and Engineering Academy and Society, Corfu Island, Greece, February 2007.

[20] S. Suh, S. Itoh, S. Aoyama, and S. Kawahito, "Column-parallel correlated multiple sampling circuits for CMOS image sensors and their noise reduction effects," Sensors, vol. 10, no. 10, pp. 9139-9154, 2010.

[21] American National Standards Institute, Photography-DirectExposing Medical and Dental Radiographic Film/Process Systems-Determination of ISO Speed and ISO Average Gradien, American National Standards Institute ISO, New York, NY, USA, 1991.

[22] F. Träger, Handbook of Lasers and Optics, Springer, Berlin, Germany, 2012.

[23] M. K. Khoury, I. Parker, and D. W. Aswad, "Acquisition of chemiluminescent signals from immunoblots with a digital single-lens reflex camera," Analytical Biochemistry, vol. 397, no. 1, pp. 129-131, 2010.

[24] T. Ang, Digital Photography: An Introduction, DK Publishing, New York, NY, USA, 4th edition, 2012.

[25] The British Journal of Photography Report. The 50 Best Photography Products of 2012. The British Journal of Photography, Apptitude Media Ltd., London, UK, 2012.

[26] R. F. Stevens and N. Davies, "Lens arrays and photography," The Journal of Photographic Science, vol. 39, pp. 199-208, 1991.

[27] R. Hooke, Micrographia: or some Physiological Descriptions of Minute Bodies made by Magnifying Glasses, The Royal Society of London, London, UK, 1st edition, 1665.

[28] P. H. Nussbaum, R. Völkel, H. P. Herzig, M. Eisner, and S. Haselbeck, "Design, fabrication and testing of microlens arrays for sensors and microsystems," Pure and Applied Optics, vol. 6, no. 6, pp. 617-636, 1997.

[29] R. A. Stern, L. Shing, N. Waltham, H. Mapson-Menard, A. Harris, and P. Pool, "EUV and soft X-ray quantum efficiency measurements of a thinned back-illuminated CMOS active pixel sensor," IEEE Electron Device Letters, vol. 32, no. 3, pp. 354356, 2011.

[30] S. N. Vasan, P. Sharma, C. N. Ionita et al., "Image acquisition, geometric correction and display of images from a $2 \times 2 \times$-ray detector array based on electron multiplying charge coupled device (EMCCD) technology," in Medical Imaging 2013: Physics of Medical Imaging, vol. 8668 of Proceedings of SPIE, Lake Buena Vista, Fla, USA, 2013.
[31] A. G. Farman and T. T. Farman, "A comparison of 18 different $\mathrm{X}$-ray detectors currently used in dentistry," Oral Surgery, Oral Medicine, Oral Pathology, Oral Radiology and Endodontics, vol. 99, no. 4, pp. 485-489, 2005.

[32] http://www.sensorgen.info/CanonEOS_5D_MkIII.html.

[33] R. G. van Silfhout and A. S. Kachatkou, "Fibre-optic coupling to high-resolution CCD and CMOS image sensors," Nuclear Instruments and Methods in Physics Research Section A: Accelerators, Spectrometers, Detectors and Associated Equipment, vol. 597, no. 2-3, pp. 266-269, 2008.

[34] H. Fan, H. L. Durko, S. K. Moore et al., "DR with a DSLR: digital radiography with a digital single-lens reflex camera," Proceedings of the Society of Photo-Optical Instrumentation Engineers, vol. 15, p. 7622, 2010.

[35] D. Uzbelger Feldman, "Real time imaging technologies, LLC, assignee. Imaging apparatus and methods," International patent application PCT/US13/49504, July 2013.

[36] W. K. Chu, C. Smith, R. Bunting, P. Knoll P, R. Wobig, and R. Thacker, "Application of the CCD camera in medical imaging," in Sensors, Cameras, and Systems for Scientific/Industrial Applications, 121, vol. 3649 of Proceedings of SPIE, San Jose, Calif, USA, January 1999.

[37] M. K. Shrout, J. Weaver, B. J. Potter, and C. F. Hildebolt, "Spatial resolution and angular alignment tolerance in radiometric analysis of alveolar bone change," Journal of Periodontology, vol. 67, no. 1, pp. 41-45, 1996.

[38] M. K. Shrout, C. M. Russell, and B. J. Potter, "Spatial resolution in radiometric analysis of enamel loss A pilot study," Oral Surgery, Oral Medicine, Oral Pathology, Oral Radiology, and Endodontics, vol. 81, no. 2, pp. 245-250, 1996.

[39] A. Wenzel, F. Haiter-Neto, and E. Gotfredsen, "Influence of spatial resolution and bit depth on detection of small caries lesions with digital receptors," Oral Surgery, Oral Medicine, Oral Pathology, Oral Radiology and Endodontology, vol. 103, no. 3, pp. 418-422, 2007.

[40] A. C. Mörner-Svalling, Digital intraoral radiography: determination of technical properties and application evaluations [Dissertation], Department of Oral Radiology and Oral and Maxillofacial Surgery, Institute of Odontology, Karolinska Institute, Stockholm, Sweden, 2002.

[41] H. S. Cho, S. G. Choi, B. S. Lee, and S. Kim, "Image quality evaluation of a highly-integrated digital X-ray imaging sensor for dental intraoral-lmaging applications," Journal of the Korean Physical Society, vol. 51, no. 1, pp. 30-34, 2007. 


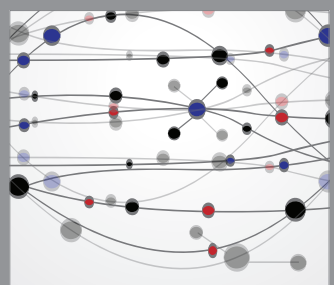

The Scientific World Journal
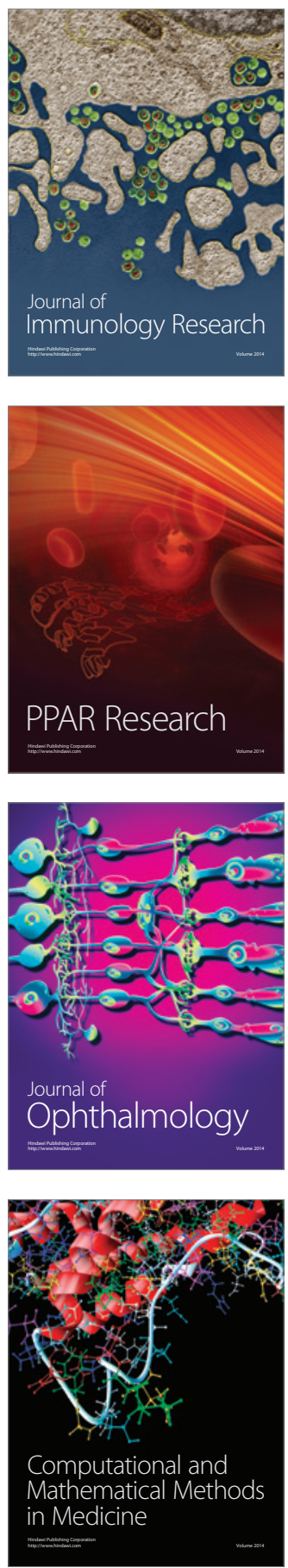

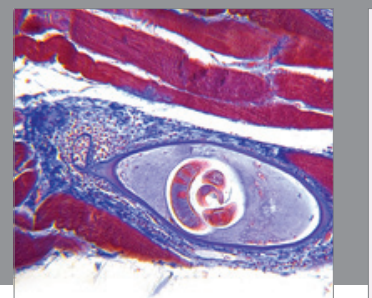

Gastroenterology

Research and Practice
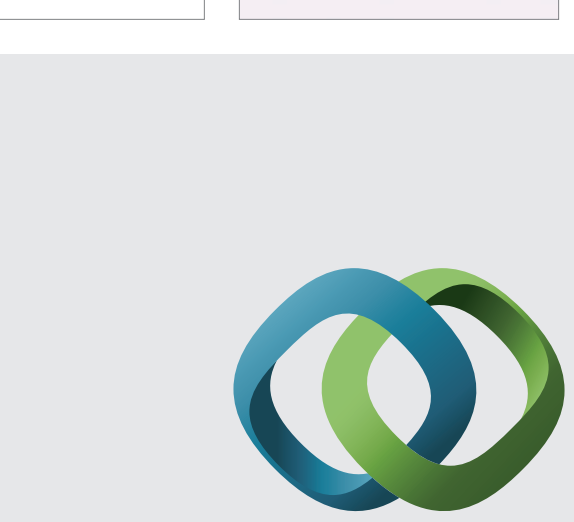

\section{Hindawi}

Submit your manuscripts at

http://www.hindawi.com
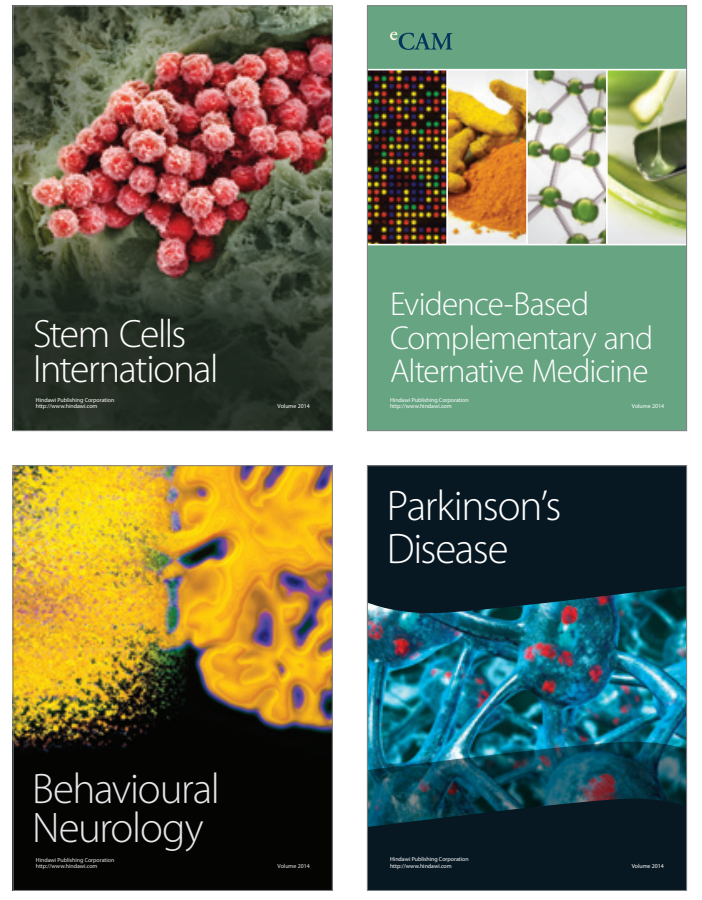
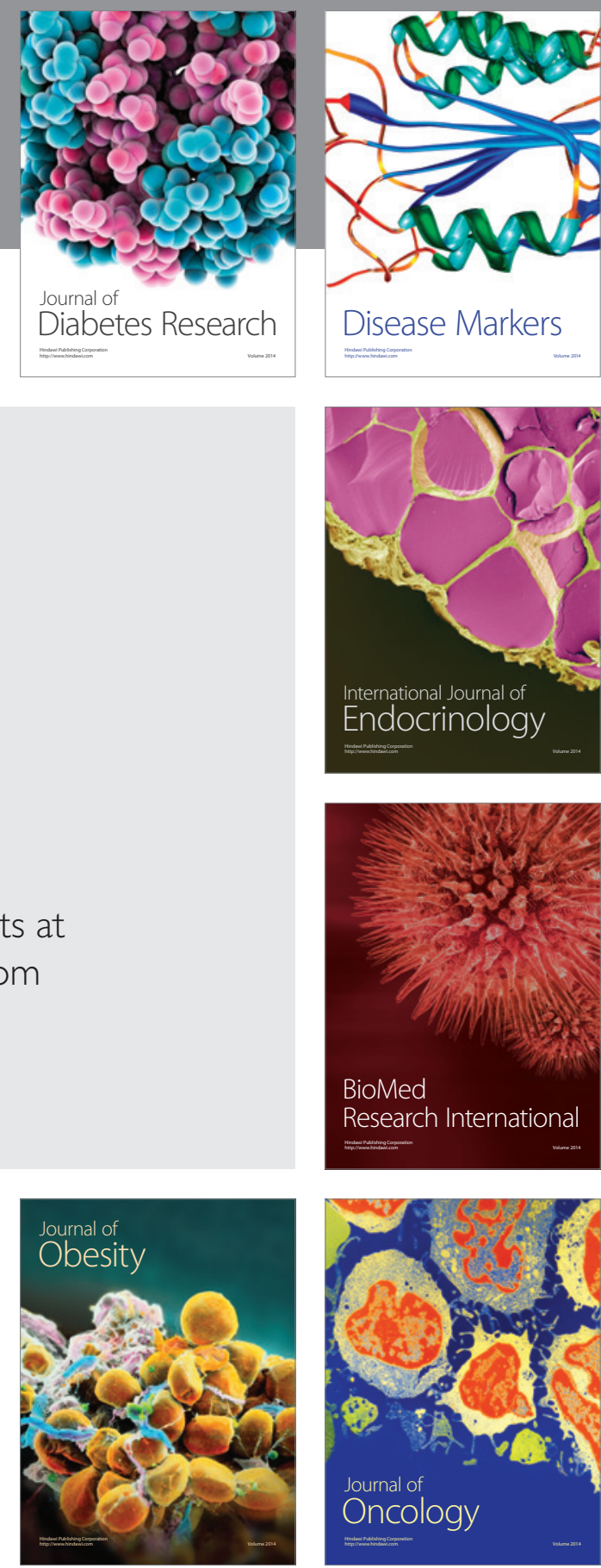

Disease Markers
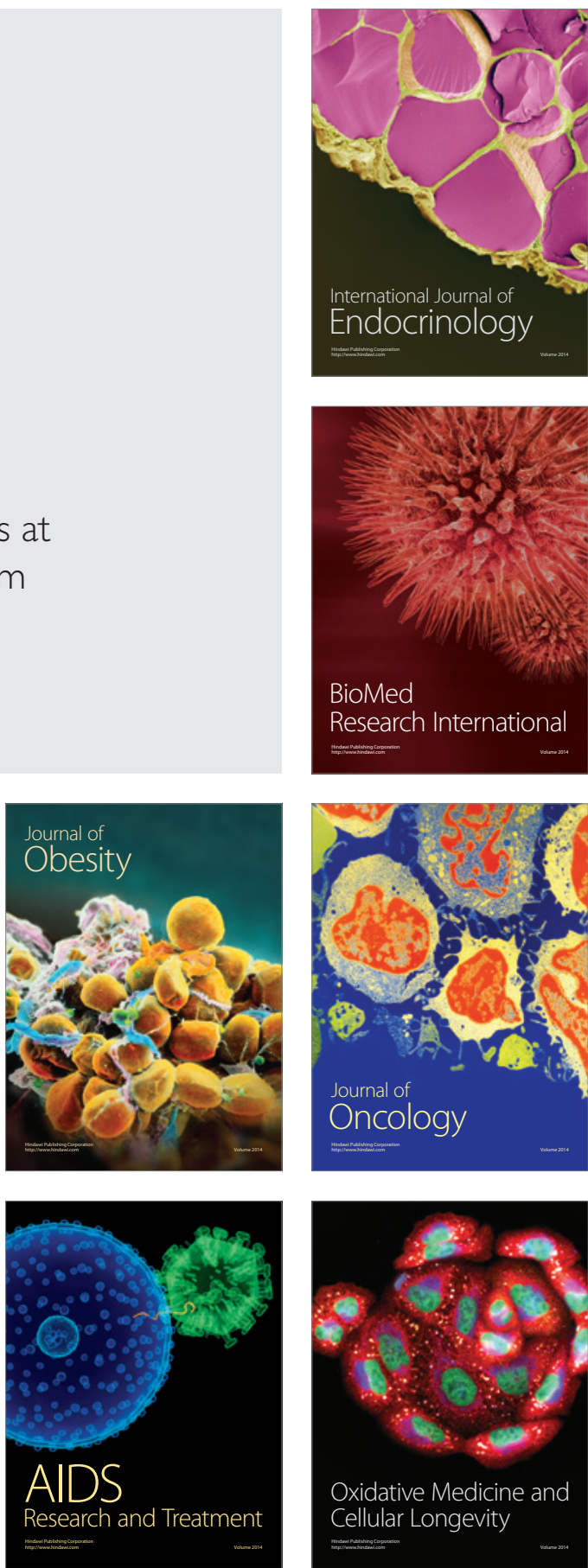